\title{
Determinacy and Large Cardinals
}

\author{
Itay Neeman*
}

\begin{abstract}
The principle of determinacy has been crucial to the study of definable sets of real numbers. This paper surveys some of the uses of determinacy, concentrating specifically on the connection between determinacy and large cardinals, and takes this connection further, to the level of games of length $\omega_{1}$.
\end{abstract}

Mathematics Subject Classification (2000). 03E55; 03E60; 03E45; 03E15.

Keywords. Determinacy, iteration trees, large cardinals, long games, Woodin cardinals.

\section{Determinacy}

Let $\omega^{\omega}$ denote the set of infinite sequences of natural numbers. For $A \subset \omega^{\omega}$ let $G_{\omega}(A)$ denote the length $\omega$ game with payoff $A$. The format of $G_{\omega}(A)$ is displayed in Diagram 1. Two players, denoted I and II, alternate playing natural numbers forming together a sequence $x=\langle x(n) \mid n<\omega\rangle$ in $\omega^{\omega}$ called a run of the game. The run is won by player I if $x \in A$, and otherwise the run is won by player II.

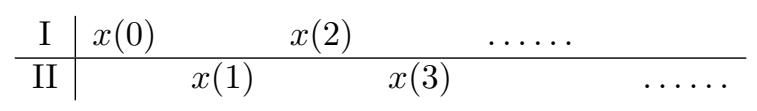

Diagram 1. The game $G_{\omega}(A)$.

A game is determined if one of the players has a winning strategy. The set $A$ is determined if $G_{\omega}(A)$ is determined. For $\Gamma \subset \mathcal{P}\left(\omega^{\omega}\right), \operatorname{det}(\Gamma)$ denotes the statement that all sets in $\Gamma$ are determined.

Using the axiom of choice, or more specifically using a wellordering of the reals, it is easy to construct a non-determined set $A$. $\operatorname{det}\left(\mathcal{P}\left(\omega^{\omega}\right)\right)$ is therefore false. On the other hand it has become clear through research over the years that $\operatorname{det}(\Gamma)$ is true if all the sets in $\Gamma$ are definable by some concrete means. Moreover $\operatorname{det}(\Gamma)$, taken as an axiom, gives rise to a rich structure theory that establishes a hierarchy of complexity on the sets in $\Gamma$, and completely answers all natural questions about the sets in each level of the hierarchy. Determinacy is therefore accepted as the natural hypothesis in the study of definable subsets of $\omega^{\omega}$.

\footnotetext{
*This material is based upon work supported by the National Science Foundation under Grant No. DMS-0094174.
} 
Definability takes increasingly liberal meanings as one progresses higher in the hierarchy of complexity. At the lower levels it is very concrete. Let $\omega^{<\omega}$ denote the set of finite sequences of natural numbers. For $s \in \omega^{<\omega}$ let $N_{s}=\left\{x \in \omega^{\omega} \mid\right.$ $x$ extends $s\}$. The sets $N_{s}\left(s \in \omega^{<\omega}\right)$ are the basic open neighborhoods in $\omega^{\omega}$. $A \subset \omega^{\omega}$ is open if it is a union of basic open neighborhoods.

$\omega^{\omega}$ with the topology defined above is isomorphic to the irrational numbers. Following standard abuse of notation in descriptive set theory we use $\mathbb{R}$ to denote $\omega^{\omega}$, and refer to its elements as reals.

A set is Borel if it can be obtained from open sets using repeated applications of complementations and countable unions. The projection of a set $B \subset \mathbb{R} \times \mathbb{R}$ is the set $\{x \in \mathbb{R} \mid(\exists y)\langle x, y\rangle \in B\}$. A set is analytic if it is the projection of the complement of an open set. A set is projective if it can be obtained from open sets using repeated applications of complementations and projections. Analyzing the logical complexity of these definitions and using diagonal arguments one can establish that $\{$ Borel sets $\} \subsetneq\{$ analytic sets $\} \subsetneq\{$ projective sets $\}$, so that these classes form a proper hierarchy.

Theorem 1.1 (Gale-Stewart [4], 1953). All open sets are determined.

Theorem 1.2 (Martin [20], 1975). All Borel sets are determined.

Theorem 1.3 (Martin [19], 1970). All analytic sets are determined.

Theorem 1.4 (Martin-Steel [22], 1985). All projective sets are determined.

Theorems 1.1 and 1.2 are theorems of ZFC, the basic system of axioms for set theory and mathematics. Theorems 1.3, 1.4, and 1.5 (below) have additional stronger assumptions known as large cardinal axioms, which are not listed here but will be discussed in Section 2. ${ }^{1}$

Recall that $\mathrm{L}(\mathbb{R})$ is the smallest model of set theory which contains all the reals and all the ordinals. It is obtained as the union $\bigcup_{\alpha \in \mathrm{ON}} \mathrm{L}_{\alpha}(\mathbb{R})$ of the hierarchy defined by the conditions: $\mathrm{L}_{0}(\mathbb{R})=\mathbb{R}$; for limit ordinals $\lambda, \mathrm{L}_{\lambda}(\mathbb{R})=\bigcup_{\alpha<\lambda} \mathrm{L}_{\alpha}(\mathbb{R})$; and for each ordinal $\alpha, \mathrm{L}_{\alpha+1}(\mathbb{R})$ consists of the sets in $\mathrm{L}_{\alpha}(\mathbb{R})$, and of all subsets of $\mathrm{L}_{\alpha}(\mathbb{R})$ which are definable over $\mathrm{L}_{\alpha}(\mathbb{R})$ by first order formulae with parameters. The third condition is the crucial one, placing a definability requirement on the sets that make it into $L(\mathbb{R}) . L(\mathbb{R})$ is constructed through a transfinite sequence of applications of this condition. Notice that the projective sets are subsumed already into $L_{1}(\mathbb{R})$, the first stage of this transfinite sequence.

Theorem 1.5 (Woodin [40], 1985). All sets of reals in $\mathrm{L}(\mathbb{R})$ are determined.

Theorems 1.1 through 1.5 establish determinacy for sets of varying levels of definability, starting from open sets which are very directly definable from real numbers, continuing with the projective sets, which are definable from open sets using existential quantifications and negations, and ending with all sets in $\mathrm{L}(\mathbb{R})$.

\footnotetext{
${ }^{1}$ The determinacy of Borel sets of course follows from the determinacy of analytic sets. The new element in Theorem 1.2 is a proof of Borel determinacy from the axioms of ZFC, without using large cardinals.
} 
More is possible, as we shall see in Section 3. The remainder of this section is devoted to consequences of determinacy.

Let $\Gamma$ be an adequate pointclass (that is a collection of subsets of $\omega^{\omega}$, closed under some basic operations, see Moschovakis [27]). The first results derived from determinacy concerned regularity properties, such as Lebesgue measurability, the Baire property, and the perfect set property. All these properties fail outside the realm of determinacy; counterexamples to each of them can be constructed easily using a wellordering of the reals. Determinacy serves as an intermediary in establishing these properties for definable sets.

Theorem 1.6 (Banach, Oxtoby [37], 1957). Assume $\operatorname{det}(\Gamma)$. Let $A \in \Gamma$. Then $A$ has the property of Baire (meaning that $A$ is either meager, or comeager on a basic open neighborhood).

Theorem 1.7 (Mycielski-Swierczkowski [29], 1964). Assume $\operatorname{det}(\Gamma)$. Then all sets in $\Gamma$ are Lebesgue measurable.

Theorem 1.8 (Davis [3], 1964). Assume $\operatorname{det}(\Gamma)$. Let $A \in \Gamma$. Then either $A$ is countable, or else A contains a perfect set.

More importantly, determinacy was seen to imply various structural properties of classes of sets within its realm. For a pointclass $\Gamma$ let $\neg \Gamma$ denote the pointclass consisting of complements of sets in $\Gamma$, and let $\exists \Gamma$ denote the pointclass consisting of projections of sets in $\Gamma$. Recall that $\boldsymbol{\Sigma}_{1}^{1}$ is the pointclass of analytic sets, $\boldsymbol{\Pi}_{n}^{1}=$ $\neg \boldsymbol{\Sigma}_{n}^{1}$, and $\boldsymbol{\Sigma}_{n+1}^{1}=\exists \boldsymbol{\Pi}_{n}^{1} . \boldsymbol{\Delta}_{n}^{1}$ is the pointclass consisting of sets which are both $\boldsymbol{\Sigma}_{n}^{1}$ and $\boldsymbol{\Pi}_{n}^{1}$. Each $\boldsymbol{\Sigma}_{n}^{1}$ set $A$ (similarly $\boldsymbol{\Pi}_{n}^{1}$ ) is definable through a string of quantifiers from an open set. The open set itself, call it $D$, is definable from a real number, coding the set $\left\{s \in \omega^{<\omega} \mid N_{s} \subset D\right\} . A$ is lightface $\Sigma_{n}^{1}$ (similarly $\Pi_{n}^{1}$ ) if the underlying real that defines it is recursive, that is computable by a Turing machine.

The boldface pointclasses were studied by analysts in the early 20 th century. Recall for example the following theorem of Kuratowski [16]: the intersection of any two $\boldsymbol{\Sigma}_{1}^{1}$ (analytic) sets $A, B \subset \mathbb{R}$ can be presented as the intersection of two $\Sigma_{1}^{1}$ sets $A^{\prime} \supset A$ and $B^{\prime} \supset B$, such that $A^{\prime} \cup B^{\prime}=\mathbb{R}$. This is today recast as a theorem about the pointclass $\Pi_{1}^{1}$. A pointclass $\Gamma$ is said to have the reduction property if for any two sets $A, B \subset \mathbb{R}$ in $\Gamma$ there are sets $A^{\prime} \subset A$ and $B^{\prime} \subset B$, both in $\Gamma$, so that $A^{\prime} \cup B^{\prime}=A \cup B$ and $A^{\prime} \cap B^{\prime}=\emptyset$. Kuratowski's theorem establishes that $\boldsymbol{\Pi}_{1}^{1}$ has the reduction property. Kuratowski also showed that $\boldsymbol{\Sigma}_{2}^{1}$ has the reduction property. This was as far up along the projective hierarchy as one could get in those days. The basic axioms of set theory, without the addition of determinacy or large cardinals, do not decide questions such as the reduction property for projective pointclasses above $\boldsymbol{\Sigma}_{2}^{1}$.

In 1967 Blackwell [2] used the determinacy of open games, Theorem 1.1 that is, to give a new proof of Kuratowski's reduction theorem. Inspired by his proof, Martin [18] and Addison-Moschovakis [1] proved that $\boldsymbol{\Pi}_{3}^{1}$ has the reduction property, assuming $\operatorname{det}\left(\boldsymbol{\Delta}_{2}^{1}\right)$.

The reduction property is a consequence of a stronger property known as the prewellordering property. Martin and Addison-Moschovakis obtained this stronger 
property, and in fact propagated it along the odd levels of the projective hierarchy, using determinacy.

A prewellorder on $A \subset \mathbb{R}$ is a relation $\preceq$ on $A$ which is transitive, reflexive, and wellfounded. The prewellorder $\preceq$ induces an equivalence relation $\sim$ on $A(x \sim y$ iff $x \preceq y \wedge y \preceq x$ ), and gives rise to a wellorder of $A / \sim$. $\preceq$ is said to belongs to a pointclass $\Gamma$ if there are two relations $Y$ and $N$, in $\Gamma$ and $\neg \Gamma$ respectively, so that for every $y \in A,\{x \mid x \preceq y\}=\{x \mid\langle x, y\rangle \in Y\}=\{x \mid\langle x, y\rangle \in N\}$. $\Gamma$ has the prewellordering property just in case that every set $A \in \Gamma$ admits a prewellorder in $\Gamma$.

Theorem 1.9 (Martin [18], Addison-Moschovakis [1], 1968). Assume projective determinacy. Then the projective pointclasses with the prewellordering (similarly reduction) property are $\boldsymbol{\Pi}_{1}^{1}, \boldsymbol{\Sigma}_{2}^{1}, \boldsymbol{\Pi}_{3}^{1}, \boldsymbol{\Sigma}_{4}^{1}, \boldsymbol{\Pi}_{5}^{1}, \ldots$

Remark 1.10. For $B \subset \mathbb{R} \times \mathbb{R}$ and $x \in \mathbb{R}$ let $B_{x}$ denote $\{y \mid\langle x, y\rangle \in B\}$. Recall that $\partial B$ is the set $\left\{x \in \mathbb{R} \mid\right.$ player I has a winning strategy in $\left.G_{\omega}\left(B_{x}\right)\right\}$. It is common to write $(\partial y) B(x, y)$, or $(\partial y)\langle x, y\rangle \in B$, for the statement $x \in$ $\partial B$. This notation is similar to the notation used for the quantifiers $(\forall y)$ and $(\exists y)$, and $(\partial y)$ too is viewed as a quantifier, giving precise meaning to the chain $(\exists y(0))(\forall y(1))(\exists(y(2)) \cdots \cdots$ of quantifiers over $\omega$. For a pointclass $\Gamma$ let $\partial \Gamma=$ $\{\partial B \mid B \in \Gamma\}$. It is easy to check that $\partial \boldsymbol{\Pi}_{n}^{1}=\boldsymbol{\Sigma}_{n+1}^{1}$ and (using determinacy) $\partial \boldsymbol{\Sigma}_{n}^{1}=\boldsymbol{\Pi}_{n+1}^{1}$. Theorem 1.9 therefore states that the pointclasses $\partial^{(n)} \boldsymbol{\Pi}_{1}^{1}, n<\omega$, all have the reduction and prewellordering properties.

Theorem 1.9 helped establish the use of determinacy as a hypothesis in the study of definable sets of reals. In particular it became standard to study $\mathrm{L}(\mathbb{R})$ using the relativization to $\mathrm{L}(\mathbb{R})$ of the assumption that all sets of reals are determined, known as the axiom of determinacy (AD) and initially advanced by Mycielski-Steinhaus [28]. The use of this assumption in $\mathrm{L}(\mathbb{R})$ is justified in retrospect by Theorem 1.5.

There has been a wealth of results on sets of reals, on structural properties of pointclasses, and on $\mathrm{L}(\mathbb{R})$, assuming determinacy. Only a couple of results, the ones which are directly relevant to this paper, are listed below. A more complete account can be found in Moschovakis [27] and in the Cabal volumes [13, 10, 11, 12].

Recall that the symbol $\boldsymbol{\delta}$ is used to denote the supremum of the ordertypes of $\Delta$ prewellorders on $\Delta$ sets.

Theorem 1.11. Assume AD. Then $\boldsymbol{\delta}_{1}^{1}$ is equal to $\omega_{1}, \boldsymbol{\delta}_{2}^{1}$ is equal to $\omega_{2}$ (Martin), and $\boldsymbol{\delta}_{3}^{1}$ is equal to $\omega_{\omega+1}$ (Martin). Much more is known, see Kechris [9] and Jackson [7].

The values of the ordinals $\boldsymbol{\delta}_{1}^{1}, \boldsymbol{\delta}_{2}^{1}$, etc. are absolute between $\mathrm{L}(\mathbb{R})$ and the true universe V. Theorem 1.11 therefore implies that $\boldsymbol{\delta}_{1}^{1}=\left(\omega_{1}\right)^{\mathrm{L}(\mathbb{R})}, \boldsymbol{\delta}_{2}^{1}=\left(\omega_{2}\right)^{\mathrm{L}(\mathbb{R})}$, and $\boldsymbol{\delta}_{3}^{1}=\left(\omega_{\omega+1}\right)^{\mathrm{L}(\mathbb{R})}$. $\omega_{1}$ is absolute between $\mathrm{L}(\mathbb{R})$ and $\mathrm{V}$, so $\boldsymbol{\delta}_{1}^{1}=\omega_{1}$. But other cardinals need not be absolute. Theorem 1.11 by itself therefore does not provide information on the cardinalities of $\boldsymbol{\delta}_{2}^{1}$ and $\boldsymbol{\delta}_{3}^{1}$. 
Theorem 1.12 (Steel-Van Wesep [38], Woodin [39]). Assume $\mathrm{AD}^{\mathrm{L}(\mathbb{R})}$. Then it is consistent (with $\mathrm{AD}^{\mathrm{L}(\mathbb{R})}$ and the axiom of choice) that $\left(\omega_{2}\right)^{\mathrm{L}(\mathbb{R})}=\omega_{2}$, and hence that $\boldsymbol{\delta}_{2}^{1}=\omega_{2}$.

Note that the statement that $\boldsymbol{\delta}_{2}^{1}=\omega_{2}$ implies a strong failure of the continuum hypothesis: not only must the continuum have size at least $\omega_{2}$, but this must be witnessed by $\boldsymbol{\Delta}_{2}^{1}$ prewellorders.

\section{Large cardinals}

An embedding $\pi: P \rightarrow M$ is elementary just in case that it preserves truth, meaning that $\varphi\left[x_{1}, \ldots, x_{k}\right]$ holds in $P$ iff $\varphi\left[\pi\left(x_{1}\right), \ldots, \pi\left(x_{k}\right)\right]$ holds in $M$, for all formulae $\varphi$ and all $x_{1}, \ldots, x_{k} \in P$. Large cardinal axioms state the existence of elementary embeddings of the universe. For example, a cardinal $\kappa$ is measurable if it is the critical point of an elementary embedding $\pi: \mathrm{V} \rightarrow M \subset \mathrm{V}$. The axiom "there exists a measurable cardinal" thus asserts the existence of a non-trivial elementary embedding acting on the entire universe.

An elementary embedding $\pi: \mathrm{V} \rightarrow M$ is $\lambda$-strong if $M$ and $\mathrm{V}$ agree to $\lambda$, that is if $M$ and $\mathrm{V}$ have the same bounded subsets of $\lambda$, and superstrong if $M$ and $\mathrm{V}$ agree to $\pi(\operatorname{crit}(\pi)) . \pi: \mathrm{V} \rightarrow M$ is $\lambda$-strong with respect to $H$ if it is $\lambda$-strong and $\pi(H) \cap \lambda=H \cap \lambda . \kappa$ is $\lambda$-strong if it is the critical point of a $\lambda$-strong embedding, and similarly for superstrength and strength with respect to $H . \kappa$ is $<\delta$-strong with respect to $H$ if it is $\lambda$-strong with respect to $H$ for each $\lambda<\delta$. Finally, and most importantly, $\delta$ is a Woodin cardinal if for every $H \subset \delta$ there is $\kappa<\delta$ which is $<\delta$-strong with respect to $H$. In the hierarchy of large cardinal axioms, the existence of Woodin cardinals lies above the existence of measurable cardinals, but well below the existence of superstrong cardinals.

Let $\pi: \mathrm{V} \rightarrow M$ be elementary. Let $\kappa=\operatorname{crit}(\pi)$ and let $\lambda<\pi(\kappa)$. The $(\kappa, \lambda)-$ extender induced by $\pi$ is the function $E: \mathcal{P}\left([\kappa]^{<\omega}\right) \rightarrow \mathcal{P}\left([\lambda]^{<\omega}\right)$ defined by $E(A)=$ $\pi(A) \cap[\lambda]^{<\omega}$. The extender $E$ codes enough of the embedding $\pi$ to reconstruct an embedding $\sigma: \mathrm{V} \rightarrow N$ with the property that $\sigma(A) \cap[\lambda]^{<\omega}=\pi(A) \cap[\lambda]^{<\omega}$ for all $A \subset[\kappa]^{<\omega}$. For sufficiently closed $\lambda$ this is enough that the $\lambda$-strength of $\pi$ implies the $\lambda$-strength of $\sigma$, and similarly for strength with respect to $H$. Thus, the existence of strong embeddings is equivalent to the existence of strong extenders, and the property of being a Woodin cardinal can be recast as a statement about the existence of certain extenders. (The point here is that extenders are sets, while embeddings are classes.)

The embedding $\sigma: \mathrm{V} \rightarrow N$ is obtained from the extender $E$ using an ultrapower construction. Very briefly, $N$ is the model $(H / \sim ; R)$ where $H=\{\langle f, a\rangle \mid a \in$ $[\lambda]^{<\omega}$ and $\left.f:[\kappa]^{\operatorname{lh}(a)} \rightarrow \mathrm{V}\right\},\langle f, a\rangle \sim\langle g, b\rangle$ iff $a^{\frown} b \in E\left(\left\{x^{\frown} y \mid f(x)=g(y)\right\}\right)$, and $[f, a] R[g, b]$ iff $a \frown b \in E(\{x \frown y \mid f(x) \in g(y)\})$. The embedding $\sigma$ is defined by the conditions $\sigma(x)=\left[c_{x}, \emptyset\right]$ where $c_{x}:[\kappa]^{0} \rightarrow \mathrm{V}$ is the function taking constant value $x$. The model $N$ is called the ultrapower of $\mathrm{V}$ by $E$, denoted $\mathrm{Ult}(\mathrm{V}, E)$, and $\sigma$ is the ultrapower embedding. 
An extender $E$ can also be derived from an embedding $\pi: Q \rightarrow M$ for $Q \neq \mathrm{V}$. The result is an extender over $Q$. In the other direction, the ultrapower of a model $Q$ by an extender $E$ with critical point $\kappa$ can be defined so long as $\left(\mathcal{P}\left([\kappa]^{<\omega}\right)\right)^{Q}=$ $\operatorname{dom}(E)$, simply by adding the restriction $f \in Q$ to the definition of $H$ above. The resulting ultrapower is denoted $\operatorname{Ult}(Q, E)$.

The process of taking ultrapowers can be iterated, and such iterations are crucial to the study of large cardinals. Their first use appeared in Kunen [15]. Kunen worked with measurable cardinals. The associated extenders can only give rise to linear iteration, and this has become the norm until the work of Martin-Steel [23], who introduced the general format of an iteration tree. This general format, which allows non-linearity, is both necessary to the study of Woodin cardinals, and non-trivial in their presence.

A tree order on an ordinal $\alpha$ is an order $T$ so that: $T$ is a suborder of $<\uparrow \alpha$; for each $\eta<\alpha$, the set $\{\xi \mid \xi T \eta\}$ is linearly ordered by $T$; for each $\xi$ so that $\xi+1<\alpha$, the ordinal $\xi+1$ is a successor in $T$; and for each limit ordinal $\gamma<\alpha$, the set $\{\xi \mid \xi T \gamma\}$ is cofinal in $\gamma$. An iteration tree $\mathcal{T}$ of length $\alpha$ on a model $M$ consists of a tree order $T$ on $\alpha$, and sequences $\left\langle M_{\xi}, j_{\zeta, \xi} \mid \zeta T \xi<\alpha\right\rangle$ and $\left\langle E_{\xi} \mid \xi+1<\alpha\right\rangle$ satisfying the following conditions:

1. $M_{0}=M$.

2. For each $\xi$ so that $\xi+1<\alpha, E_{\xi}$ is an extender of $M_{\xi}$.

3. $M_{\xi+1}=\operatorname{Ult}\left(M_{\zeta}, E_{\xi}\right)$ and $j_{\zeta, \xi+1}: M_{\zeta} \rightarrow M_{\xi+1}$ is the ultrapower embedding, where $\zeta$ is the $T$-predecessor of $\xi+1$. It is implicit in this condition that $\mathcal{P}\left(\left[\operatorname{crit}\left(E_{\xi}\right)\right]^{<\omega}\right)$ must be the same in $M_{\zeta}$ and $M_{\xi}$, so that the ultrapower makes sense.

4. For limit $\lambda<\alpha, M_{\lambda}$ is the direct limit of the system $\left\langle M_{\zeta}, j_{\zeta, \xi} \mid \zeta T \xi T \lambda\right\rangle$, and $j_{\zeta, \lambda}: M_{\zeta} \rightarrow M_{\lambda}$ for $\zeta T \lambda$ are the direct limit embeddings.

5. The remaining embeddings $j_{\zeta, \xi}$ for $\zeta T \xi<\alpha$ are obtained through composition.

An iteration tree is linear if for every $\xi$, the $T$-predecessor of $\xi+1$ is $\xi$.

A branch through an iteration tree $\mathcal{T}$ is a set $b$ which is linearly ordered by $T$. The branch is cofinal if $\sup (b)=\operatorname{lh}(\mathcal{T})$. The branch is maximal if either $\sup (b)=\operatorname{lh}(\mathcal{T})$ or else $b \neq\{\xi \mid \xi T \sup (b)\}$. The direct limit along $b$, denoted $M_{b}^{\mathcal{T}}$ or simply $M_{b}$, is the direct limit of the system $\left\langle M_{\xi}, j_{\zeta, \xi} \mid \zeta T \xi \in b\right\rangle . i_{b}^{\mathcal{T}}: M \rightarrow M_{b}$ is the direct limit embedding of this system. The branch $b$ is called wellfounded just in case that the model $M_{b}$ is wellfounded.

Theorem 2.1 (Martin-Steel [23]). Let $M$ be a countable elementary substructure of a rank initial segment $\mathrm{V}$, and let $\pi: M \rightarrow \mathrm{V}_{\nu}$ be elementary. Let $\mathcal{T}$ be a countable iteration tree on $M$. Then there is a maximal branch $b$ through $\mathcal{T}$, and an embedding $\sigma: M_{b} \rightarrow \mathrm{V}_{\nu}$, so that $\pi=\sigma \circ i_{b}^{\mathcal{T}}$. (A branch $b$ whose direct limit can be embedded into $\mathrm{V}_{\nu}$ in this way is called realizable.) 
Let $M$ be a model of ZFC. In the (full, length $\omega_{1}+1$ ) iteration game on $M$ players "good" and "bad" collaborate to construct an iteration tree $\mathcal{T}$ of length $\omega_{1}^{\mathrm{V}}+1$ on $M$. "bad" plays all the extenders, and determines the $T$-predecessor of $\xi+1$ for each $\xi$. "good" plays the branches $\{\zeta \mid \zeta T \lambda\}$ for limit $\lambda$, thereby determining the direct limit model $M_{\lambda}$. Note that "good" is also responsible for the final move, which determines $M_{\omega_{1}^{\mathrm{v}}}$.

If ever a model along the tree is reached which is illfounded then "bad" wins. Otherwise "good" wins. $M$ is (fully) iterable if "good" has a winning strategy in this game. An iteration strategy for $M$ is a winning strategy for the good player in the iteration game on $M$.

Notice that if Theorem 2.1 could be strengthened to state that the realizable branch is unique, then repeated applications of the theorem (including a final application over $\mathrm{V}^{\operatorname{col}\left(\omega, \omega_{1}\right)}$ to obtain a branch through a tree of length $\left.\omega_{1}^{\mathrm{V}}\right)$ would demonstrate that countable elementary substructures of rank initial segments of $\mathrm{V}$ are iterable. This observation is the key to many of the known iterability proofs, but unfortunately uniqueness fails beyond certain large cardinals. A general proof of iterability would be a great step forward in the study of large cardinals.

A (fine structural) inner model is a model of the form $M=\mathrm{L}_{\alpha}(\vec{E})$, that is the smallest model of set theory containing the ordinals below $\alpha$ and closed under comprehension relative to $\vec{E}$, where $\vec{E}$ is a sequence of extenders, over $M$ or over initial segments of $M$, satisfying certain coherence requirements. (There are various ways to structure the sequences. For precise definitions see Mitchell-Steel [26] or Zeman [42].) $M=\mathrm{L}_{\alpha}(\vec{E})$ is an initial segment of $N=\mathrm{L}_{\beta}(\vec{F})$ just in case that $\alpha \leq \beta$ and $\vec{E}$ is an initial segment of $\vec{F}$. Since the extenders in $\vec{E}$ may be extenders not over $M$ but over strict initial segments of $M$, an iteration tree on $M$ may involve dropping to initial segments, that is applying an extender in $M_{\xi}$ to an initial segment of $M_{\zeta}$. In such cases the embedding $j_{\zeta, \xi+1}$ acts on an initial segment of $M_{\zeta}$.

The following fact is the key to the use of iteration trees in the study of inner models:

Fact 2.2 (Comparison). Let $M$ and $N$ be countable inner models. Suppose that $M$ and $N$ are both iterable. Then there are iteration trees $\mathcal{T}$ and $\mathcal{U}$ of countable lengths on $M$ and $N$ respectively, leading to final models $M^{*}$ and $N^{*}$, so that at least one of the following conditions holds:

1. $M^{*}$ is an initial segment of $N^{*}$ and there are no drops on the branch of $\mathcal{T}$ leading from $M$ to $M^{*}$.

2. $N^{*}$ is an initial segment of $M^{*}$ and there are no drops on the branch of $\mathcal{U}$ leading from $N$ to $N^{*}$.

The iteration trees $\mathcal{T}$ and $\mathcal{U}$ witnessing Fact 2.2 are constructed inductively. Suppose the construction reached models $M_{\xi}$ on $\mathcal{T}$ and $N_{\xi}$ on $\mathcal{U}$. If the extender sequences of $M_{\xi}$ and $N_{\xi}$ agree, meaning that they are equal or that one is a strict initial segment of the other, then the construction is over and one of conditions (1) and (2) in Fact 2.2 holds. If the sequences do not agree, let $\rho$ be least so that 
the extender sequences of $M_{\xi}$ and $N_{\xi}$ disagree on the $\rho$ th extender. Set $E_{\xi}$ to be the $\rho$ th extender on the sequence of $M_{\xi}$, and use this assignment to continue the construction of $\mathcal{T}$, applying $E_{\xi}$ to $M_{\zeta}$ for the smallest possible $\zeta$, to give rise to $M_{\xi+1}$. Continue $\mathcal{U}$ similarly using the $\rho$ th extender on the sequence of $N_{\xi}$. These assignments determine the parts of $\mathcal{T}$ and $\mathcal{U}$ corresponding to the bad player's moves in the iteration game. Using the assumption that $M$ and $N$ are iterable, fix iteration strategies $\Sigma$ and $\Lambda$ for the two models, and let these strategies determine the remaining elements of $\mathcal{T}$ and $\mathcal{U}$, namely the branches to be used at limit stages.

It is one of the great discoveries of inner model theory that the process described above, of repeatedly forming ultrapowers by disagreeing extenders, terminates, leading therefore to models which are lined-up with their extender sequences in complete agreement. The discovery was first made by Kunen [15] in the context of a single measurable cardinal, where linear iterations suffice. Mitchell [24, 25] developed the framework for models with many measurable cardinals, still using linear iterations. Martin-Steel [22, 23] discovered that in the context of Woodin cardinals the more general (non-linear) iteration trees are both needed and sufficient. Mitchell-Steel [26] used iteration trees, fine structure (see Jensen [8]), and several additional ideas to develop inner models for Woodin cardinal, and reach Fact 2.2 as stated above.

The following folklore claim illustrates a simple application of the comparison process. An inner model $M$ is called a minimal model for a sentence $\theta$ if $M$ satisfies $\theta$ and no strict initial segment of $M$ satisfies $\theta$.

Claim 2.3. Let $M$ and $N$ be minimal countable inner models for the same sentence $\theta$. Suppose that both $M$ and $N$ are iterable. Then $M$ and $N$ have the same theory.

Proof sketch. Compare $M$ and $N$, that is form $\mathcal{T}$ and $\mathcal{U}$ leading to models $M^{*}$ and $N^{*}$ which are in complete agreement, using Fact 2.2. Neither one of $M^{*}$ and $N^{*}$ can be a strict initial segment of the other, since otherwise the longer of the two will have a strict initial segment satisfying $\theta . M^{*}$ and $N^{*}$ must therefore be equal. Similar reasoning shows that there can be drops on either side of the comparison. Using the elementarity of the iteration embeddings (from $M$ to $M^{*}$ along $\mathcal{T}$, and from $N$ to $N^{*}$ along $\mathcal{U}$ ) it follows that $M$ has the same theory as $M^{*}$ and $N$ has the same theory as $N^{*}$. Since $M^{*}=N^{*}, M$ and $N$ have the same theory.

An inner model $M$ is a sharp if its extender sequence has a final element, $E_{\mathrm{top}}^{M}$, and $E_{\text {top }}^{M}$ is an extender over $M$. For a sharp $M$ let $M^{*}$ be the result of iterating $E_{\text {top }}^{M}$ through the countable ordinals, that is set $M^{\prime}$ equal to the final model of the iteration tree $\mathcal{T}$ defined by the condition $E_{\xi}=j_{0, \xi}\left(E_{\text {top }}^{M}\right)$ and the $T$-predecessor of $\xi+1$ is $\xi$ for all $\xi<\omega_{1}$, and let $M^{*}=M^{\prime} \| \omega_{1}$. The set $I=\left\{j_{0, \xi}\left(\operatorname{crit}\left(E_{\text {top }}^{M}\right)\right) \mid \xi<\right.$ $\left.\omega_{1}\right\}$ is club in $\omega_{1}$. The ordinals in $I$ are indiscernibles for $M^{*}$, in the sense that for any formula $\varphi$, and any increasing sequences $\left\{\alpha_{1}, \ldots, \alpha_{k}\right\}$ and $\left\{\beta_{1}, \ldots, \beta_{k}\right\}$ in $[I]^{k}, M^{*} \models \varphi\left[\alpha_{1}, \ldots, \alpha_{k}\right]$ iff $M^{*} \models \varphi\left[\beta_{1}, \ldots, \beta_{k}\right]$. The theory of $k$ indiscernibles for $M$, denoted $\operatorname{Th}_{k}(M)$, is the set of formulae $\varphi$ so that $M^{*} \models \varphi\left[\alpha_{1}, \ldots, \alpha_{k}\right]$ for some (equivalently all) $\left\{\alpha_{1}, \ldots, \alpha_{k}\right\} \in[I]^{k}$.

An argument similar to that of Claim 2.3 shows that if $M$ and $N$ are both minimal iterable sharps for the same sentence $\theta$, then $\operatorname{Th}_{k}(M)=\operatorname{Th}_{k}(N)$. The 
join $\oplus_{k<\omega} \operatorname{Th}_{k}(M)$ is called the sharp for $\theta$. The sharp for the sentence "there are $n$ Woodin cardinals" is called the sharp for $n$ Woodin cardinals. The sharp for a tautology is denoted $0^{\sharp}$. It codes a club of indiscernibles for $\mathrm{L}$.

The existence of $0^{\sharp}$ follows from the existence of a measurable cardinal. But in general the existence of the sharp for $\theta$ does not follow directly from the existence of large cardinals in $\mathrm{V}$. The sharp also require iterability, which is used in an essential way through the appeal to the comparison process in the proof of Claim 2.3. At the level of finitely many Woodin cardinals iterability can be obtained using Theorem 2.1 and additional arguments on the uniqueness of realizable branches, so that the existence of the sharp for $n$ Woodin cardinals follows from the existence in $\mathrm{V}$ of $n$ Woodin cardinals and a measurable cardinal above them.

It was noted in Section 1 that proofs of determinacy for pointclasses from $\mathbf{\Pi}_{1}^{1}$ onward require large cardinal axioms. To be specific, a proof of determinacy for the pointclass $\Pi_{1}^{1}$ (Theorem 1.3) requires roughly the existence of a measurable cardinal, a proof of determinacy for the pointclass $\boldsymbol{\Pi}_{n+1}^{1}$ (Theorem 1.4) requires roughly the existence of $n$ Woodin cardinals and a measurable cardinal above them, and a proof of determinacy for the pointclass of all sets in $L(\mathbb{R})$ (Theorem 1.5) requires roughly the existence of $\omega$ Woodin cardinals and a measurable cardinal above them. But this is only the beginning of the connection between these pointclasses and Woodin cardinals.

Recall that a set $A$ is $\alpha-\Pi_{1}^{1}$ if there is a sequence $\left\langle A_{\xi} \mid \xi<\alpha\right\rangle$ of $\Pi_{1}^{1}$ sets so that $x \in A$ iff the least $\xi$ so that $x \notin A_{\xi} \vee \xi=\alpha$ is odd. (The hierarchy generated by this definition is the difference hierarchy on $\boldsymbol{\Pi}_{1}^{1}$ sets. Note for example that for $\alpha=2$ the condition states simply that $A=A_{0}-A_{1}$.) The set $A$ is (lightface) $\alpha-\Pi_{1}^{1}$ if the underlying code for the sequence $\left\langle A_{\xi} \mid \xi<\alpha\right\rangle$ is recursive. $A$ is $<\omega^{2}-\Pi_{1}^{1}$ if it is $\alpha-\Pi_{1}^{1}$ for some $\alpha<\omega^{2}$.

Theorem 2.4 (Martin [21]). Let $B_{i}(i<\omega)$ be a recursive enumeration of the $<\omega^{2}-\Pi_{1}^{1}$ sets. Then each of $0^{\sharp}$ and $\left\{i \mid\right.$ player I has a winning strategy in $\left.G_{\omega}\left(B_{i}\right)\right\}$ is recursive in the other.

Theorem 2.4 provides a very tight connection between a large cardinal object, $0^{\sharp}$, and infinite games. For every formula $\varphi$ there is a $<\omega^{2}-\Pi_{1}^{1}$ set $B$ so that $\varphi$ belongs to $0^{\sharp}$ iff I wins $G_{\omega}(B)$, and conversely (for every $B$ there is $\varphi$ ).

Theorem 2.5. Let $B_{i}(i<\omega)$ be a recursive enumeration of the $\partial^{(n)}\left(<\omega^{2}-\Pi_{1}^{1}\right)$ sets. Then the sharp for $n$ Woodin cardinals and $\{i \mid$ player I has a winning strategy in $\left.G_{\omega}\left(B_{i}\right)\right\}$ are each recursive in the other.

Theorem 2.5 generalizes Theorem 2.4 to $n>0$. It has two directions. The first states that membership in the sharp for $n$ Woodin cardinals is equivalent to winning a $\partial^{(n)}\left(<\omega^{2}-\Pi_{1}^{1}\right)$ game. This follows from the results of Martin-Steel [23]. Essentially they show that iterability (or more precisely the ability to survive through the comparison process) for minimal sharps for $n$ Woodin cardinals, can be expressed as a $\partial^{(n)}\left(<\omega^{2}-\Pi_{1}^{1}\right)$ statement. The other direction of Theorem 2.5 states that sharps for $n$ Woodin cardinals can discern which player wins a $\partial^{(n)}\left(<\omega^{2}-\Pi_{1}^{1}\right)$ game. This direction follows from the determinacy proof in Neeman [30, 32]. The 
proof reduces the quantifiers involved in the $\partial^{(n)}\left(<\omega^{2}-\Pi_{1}^{1}\right)$ set to an iteration game on any model which has a sharp for $n$ Woodin cardinals. The reduction takes place inside the model, and the model can tell which player in the $\partial^{(n)}\left(<\omega^{2}-\Pi_{1}^{1}\right)$ game is matched to the good player in the iteration game. Since the sharp is iterable, this player wins the $\partial^{(n)}\left(<\omega^{2}-\Pi_{1}^{1}\right)$ game.

Theorem 2.5 is an indication of the close connections between the study of inner models for Woodin cardinals and the study of projective pointclasses. The connections are tight enough that inner models can be used directly in the study of projective pointclasses, and further up in the study of $\mathrm{L}(\mathbb{R})$ under determinacy.

Theorem 2.6 (Neeman-Woodin, see [30]). Determinacy for all $\boldsymbol{\Pi}_{n+1}^{1}$ sets implies determinacy for all sets in the larger pointclass $\partial^{(n)}\left(<\omega^{2}-\Pi_{1}^{1}\right)$.

Theorem 2.7 (Hjorth [6]). Work in $\mathrm{L}(\mathbb{R})$ assuming AD. Let $\preceq$ be a $\partial\left(\alpha-\Pi_{1}^{1}\right)$ prewellorder with $\alpha<\omega \cdot k$. Then the ordertype of $\preceq$ is smaller than $\omega_{k+1}$.

Theorem 2.8 (Neeman, Woodin, see [36]). Assume $\mathrm{AD}^{\mathrm{L}(\mathbb{R})}$. Then it is consistent (with $\mathrm{AD}^{\mathrm{L}(\mathbb{R})}$ and the axiom of choice) that $\boldsymbol{\delta}_{3}^{1}=\omega_{2}$.

Theorem 2.6 for $n=0$ is a combination of the work of Harrington [5], who obtained $0^{\sharp}$ and its relativized versions for all reals from $\Pi_{1}^{1}$ determinacy, and Martin, who obtained $<\omega^{2}-\Pi_{1}^{1}$ determinacy from the sharps. At higher levels Woodin obtained sharps for $n$ Woodin cardinals from $\boldsymbol{\Pi}_{n+1}^{1}$ determinacy and Neeman [30] obtained $\partial^{(n)}\left(<\omega^{2}-\Pi_{1}^{1}\right)$ determinacy from these sharps. Theorem 2.6 had already been proved for odd $n$ by Kechris-Woodin [14], using methods which are purely descriptive set theoretic. For even $n$ the only known proofs involve large cardinals.

Hjorth [6] proved Theorem 2.7 by embedding a given $\partial\left(<\omega \cdot k-\Pi_{1}^{1}\right)$ prewellorder into a directed system of iterates of a sharp for one Woodin cardinal, and proving that the rank of the directed system is smaller than $\omega_{k+1}$. Again, the proof is closely tied up with large cardinals and iteration trees, even though the result is purely descriptive set theoretic.

Theorem 2.8 is proved by collapsing $\omega_{\omega}$ to $\omega_{1}$ over $\mathrm{L}(\mathbb{R})$, so that $\left(\omega_{\omega+1}\right)^{\mathrm{L}(\mathbb{R})}$, which is equal to $\boldsymbol{\delta}_{3}^{1}$ by Theorem 1.11, becomes $\omega_{2}$ of the generic extension. The forcing to collapse $\omega_{\omega}$ involves an ultrafilter on $\left[\mathcal{P}_{\omega_{1}}\left(\omega_{\omega}\right)\right]^{<\omega_{1}}$, and the construction of this ultrafilter uses a directed system of iterates of fine structural inner models with Woodin cardinals.

\section{Larger cardinals, longer games}

For $\alpha<\omega_{1}$ and $B \subset \mathbb{R}^{\alpha}$ let $G_{\omega \cdot \alpha}(B)$ denote the length $\omega \cdot \alpha$ game with payoff $B$. Players I and II alternate playing natural numbers in the format of Diagram 2, taking $\omega \cdot \alpha$ moves to produce together a sequence $r=\langle r(\xi)| \xi\langle\omega \cdot \alpha\rangle$ in $\omega^{\omega \cdot \alpha}$. The sequence $r$ may be viewed as an element of $\left(\omega^{\omega}\right)^{\alpha}=\mathbb{R}^{\alpha}$. If $r$ belongs to $B$ then player I wins, and otherwise player II wins. 


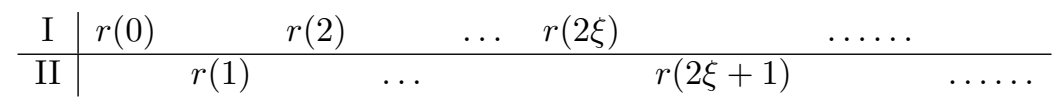

Diagram 2. General format of a transfinite game.

Determinacy for all length $\omega$ games with payoff in $\partial^{(n)}\left(<\omega^{2}-\Pi_{1}^{1}\right)$ is easily seen to be the same as determinacy for all games of length $\omega \cdot(n+1)$ with payoff in $<\omega^{2}-$ $\Pi_{1}^{1}$. Theorem 2.5 and the part of Theorem 2.6 dealing with a proof of determinacy from sharps can therefore be rephrased as follows:

Theorem 3.1. Let $B_{i}(i<\omega)$ be a recursive enumeration of all the $<\omega^{2}-\Pi_{1}^{1}$ sets. Suppose that there is an iterable sharp for $n$ Woodin cardinals. Then all length $\omega \cdot(n+1)$ games with payoff in $<\omega^{2}-\Pi_{1}^{1}$ are determined. Moreover, the sharp for $n$ Woodin cardinals and $\left\{i \mid\right.$ player I wins $\left.G_{\omega \cdot(n+1)}\left(B_{i}\right)\right\}$ are each recursive in the other.

The same precise connection between large cardinals and determinacy can be obtained higher up. Theorems 3.2, 3.3, and 3.4 below give several markers along the hierarchies of large cardinals and determinacy, progressively moving upward on both.

Theorem 3.2 (Neeman, Woodin, see [33, Chapter 2]). Let $\alpha$ be a countable ordinal. Let $B_{i}(i<\omega)$ be a recursive enumeration of all the $<\omega^{2}-\Pi_{1}^{1}$ subsets of $\mathbb{R}^{1+\alpha}$. Suppose that there is an iterable sharp for $\alpha$ Woodin cardinals. Then all length $\omega \cdot(1+\alpha)$ games with payoff in $<\omega^{2}-\Pi_{1}^{1}$ are determined. Moreover, the sharp for $\alpha$ Woodin cardinals and $\left\{i \mid\right.$ player I wins $\left.G_{\omega \cdot(1+\alpha)}\left(B_{i}\right)\right\}$ are each recursive in the other.

For $B \subset \omega^{<\omega_{1}}$ let $G_{\text {adm }}(B)$ be the following game: Players I and II alternate natural numbers as in Diagram 2, continuing until they reach the first ordinal $\alpha$ so that $\mathrm{L}_{\alpha}[r(\xi) \mid \xi<\alpha]$ is admissible. At that point the game ends. Player I wins if $\langle r(\xi) \mid \xi<\alpha\rangle \in B$, and otherwise player II wins.

The run $\langle r(\xi) \mid \xi<\alpha\rangle$ has the property that for every $\beta<\alpha, \mathrm{L}_{\beta}[r(\xi) \mid \xi<\beta]$ is not admissible. Using this property the run can be coded by a real in a canonical, uniform manner. The payoff set $B$ is said to be $\Gamma$ in the codes just in case that the set of real codes for sequences in $B$ belongs to $\Gamma$.

$G_{\text {adm }}(B)$ is a game of variable countable length. Its runs are countable, but the length of a particular run depends on the moves made during the run. Each of the players can force the length of the run to be greater than any fixed countable ordinal $\alpha$, and the determinacy of $G_{\mathrm{adm}}(B)$ for all $B$ in $\left\langle\omega^{2}-\Pi_{1}^{1}\right.$ implies the determinacy of $G_{\alpha}(B)$ for all $B$ in $<\omega^{2}-\Pi_{1}^{1}$, for each countable $\alpha$.

The Mitchell order on extenders is the order $E \triangleleft F$ iff $E \in \operatorname{Ult}(V, F)$. The Mitchell order of a cardinal $\kappa$ is the ordertype of the restriction of $\triangleleft$ to extenders with critical point $\kappa$.

\footnotetext{
${ }^{2}$ Recursiveness here is relative to a code for $\alpha$.
} 
Theorem 3.3 (Neeman [34]). Let $B_{i}(i<\omega)$ be a recursive enumeration of the subsets of $\omega^{<\omega_{1}}$ which are $<\omega^{2}-\Pi_{1}^{1}$ in the codes. Suppose that there is an iterable sharp for the statement "there is a cardinal $\kappa$ which is a limit of Woodin cardinals and has Mitchell order $\kappa^{++}$. Then the games $G_{\mathrm{adm}}(B)$ are determined for all $B$ which are $<\omega^{2}-\Pi_{1}^{1}$ in the codes. Moreover the sharp and the real $\{i \mid$ player I wins $\left.G_{\mathrm{adm}}\left(B_{i}\right)\right\}$ are each recursive in the other.

For $B \subset \omega^{<\omega_{1}}$ let $G_{\text {local }}(\mathrm{L}, B)$ be the following game: Players I and II alternate natural numbers as in Diagram 2, continuing until they reach the first $\alpha>\omega$ so that $\alpha$ is a cardinal in $\mathrm{L}[r(\xi) \mid \xi<\alpha]$. At that point the game ends. Player I wins if $\langle r(\xi) \mid \xi<\alpha\rangle \in B$. Otherwise player II wins. $G_{\text {local }}(\mathrm{L}, B)$ is a game ending at $\omega_{1}$ in $\mathrm{L}$ of the play. It too is a game of variable countable length.

A code for a run $\langle r(\xi) \mid \xi<\alpha\rangle$ of $G_{\text {local }}(\mathrm{L}, B)$ is simply a pair $\langle w, x\rangle$ where $w$ is a wellorder of $\omega$ of ordertype $\alpha, x \in \omega^{\omega}$, and for each $n, x(n)$ is equal to $r(\xi)$ where $\xi$ is the ordertype of $n$ in $w$. These codes belong to $\mathcal{P}(\omega \times \omega) \times \omega^{\omega}$, which is isomorphic to $\omega^{\omega}$. As before, $B$ is said to be $\Gamma$ in the codes just in case that the set of codes for sequences in $B$ belongs to $\Gamma$.

Theorem 3.4 (Neeman [33, Chapter 7]). Let $B_{i}(i<\omega)$ be a recursive enumeration of the subsets of $\omega^{<\omega_{1}}$ which are $\partial\left(<\omega^{2}-\Pi_{1}^{1}\right)$ in the codes. Suppose that there is an iterable sharp for the statement "there is a Woodin cardinal which is also a limit of Woodin cardinals". Then the games $G_{\text {local }}(\mathrm{L}, B)$ are determined for all $B$ which are $\partial\left(<\omega^{2}-\Pi_{1}^{1}\right)$ in the codes. Moreover the sharp and the real $\{i \mid$ player I wins $\left.G_{\text {local }}\left(\mathrm{L}, B_{i}\right)\right\}$ are each recursive in the other.

Remark 3.5. Theorem 3.4 has an interesting corollary, due to Woodin: Suppose that there is an iterable sharp for a Woodin limit of Woodin cardinals. Then it is consistent that all ordinal definable games of length $\omega_{1}$ are determined. The model witnessing this is of the form $M=\mathrm{L}[x(\xi) \mid \xi<\gamma]$ where $\gamma=\omega_{1}^{M}$, and the strategies witnessing determinacy in this model are obtained through uses of Theorem 3.4 on games ending at $\omega_{1}$ in L of the play. For a complete proof of the corollary see Neeman [33, 7F.13-15].

Remark 3.6. There is another interesting game that comes up in the proof of Theorem 3.4. For a partial function $f: \mathbb{R} \rightarrow \omega$ and a set $B \subset \omega^{<\omega_{1}}$ let $G_{\text {cont }}(f, B)$ be the following game: Players I and II alternate natural numbers as in Diagram 2. In addition, after each block of $\omega$ moves they write a natural numbers on a "side board". Let $x_{\alpha}=\langle r(\omega \cdot \alpha+i) \mid i<\omega\rangle$ be the $\alpha$ th block of moves. The natural number they write following this block is $n_{\alpha}=f\left(x_{\alpha}\right)$. They continue playing until reaching the first $\alpha$ so that $x_{\alpha} \notin \operatorname{dom}(f)$ or $n_{\alpha} \in\left\{n_{\beta} \mid \beta<\alpha\right\}$ (meaning that the natural number written after block $\alpha$ is a repetition of a number written previously). At that point the game ends, player I wins if $\langle r(\xi)| \xi\langle\omega \cdot \alpha+\omega\rangle \in B$, and player II wins otherwise. The large cardinal strength of determinacy for these games is roughly a cardinal $\kappa$ which is $\delta+1$-strong for some Woodin cardinal $\delta>\kappa$ (see Neeman [33, Chapter 3]), and the determinacy proof for these games is a precursor to the use of extenders overlapping Woodin cardinals in the proof of Theorem 3.4. 
Determinacy in Theorems 3.2, 3.3, and 3.4 is proved by reducing the long game to an iteration game on the given model. The reduction, which uses the large cardinals of the model, matches one of the players in the long game to the good player in the iteration game. In effect it converts the iteration strategy for the model into a winning strategy for this player in the long game. Determinacy therefore rests on the existence of iterable models; the existence of large cardinals by itself is not directly sufficient.

In the case of Theorems 3.2 and 3.3, the long game is reduced to an iteration game of a specific format, involving only linear compositions of iteration trees of length $\omega$. The fact that the good player can win games of this format, on countable model which embed into rank initial segments of $\mathrm{V}$, follows directly from Theorem 2.1. The determinacy proved in Theorems 3.2 and 3.3 therefore follows from just the assumptions of large cardinals in $\mathrm{V}: \alpha$ Woodin cardinals and a measurable cardinal above them in the case of Theorem 3.2, and a measurable cardinal above a cardinal $\kappa$ so that $o(\kappa)=\kappa^{++}$and $\kappa$ is a limit of Woodin cardinals in the case of Theorem 3.3.

The iteration game generated by the proof of Theorem 3.4 is as complicated as the full iteration game, and Theorem 2.1 by itself is not enough to produce a winning strategy for the good player in this game. Still, by Neeman [31], the existence of an iterable model satisfying the large cardinal assumptions of Theorem 3.4 follows from the existence of the large cardinals, a Woodin limit of Woodin cardinals and a measurable cardinal above it, in V.

Theorems 3.2, 3.3, and 3.4 extend the precise connection between determinacy and inner models to levels of games of variable countable lengths, and Woodin limits of Woodin cardinals. It is generally believed that the large cardinal hierarchy is rich enough to calibrate the strength of all natural statements. Could determinacy provide a rich enough hierarchy to match the full extent of the large cardinal hierarchy? If not, how far does determinacy reach? How far does the hierarchy of long games reach? We are very far from answers to these questions.

Let $\theta$ be a large cardinal assumption at or below the existence of a superstrong cardinal. (Beyond the level of superstrong cardinals there are problems with the comparison process and Claim 2.3.) The comparison process provides the best clues in the search for long games strong enough to match $\theta$ in the sense of Theorems 3.2, 3.3, and 3.4: If a particular format of long games subsumes the iteration games appearing in the comparison of minimal models of $\theta$, then the associated game quantifier is strong enough to define the sharp for $\theta$.

The following format is strong enough to subsume the full iteration game, and therefore all iteration games appearing in all comparisons of all inner models up to superstrong cardinals. Let $\mathcal{L}^{+}$denote the language of set theory with an added unary relation symbol $\dot{r}$, and let $\varphi(\alpha, \beta)$ be a formula in $\mathcal{L}^{+}$. Define $G_{\text {club }, 2}(\varphi)$ to be the following game: Players I and II alternate playing $\omega_{1}$ natural numbers in the manner of Diagram 2, producing together a sequence $\langle r(\xi)| \xi\left\langle\omega_{1}\right\rangle$ in $\omega^{\omega_{1}}$. If there is a club $C \subset \omega_{1}$ so that $\left\langle\mathrm{L}_{\omega_{1}}[r], r\right\rangle \models \varphi[\alpha, \beta]$ for all $\alpha<\beta$ both in $C$ then player I wins, and otherwise player II wins. (A quick word on notation: $r$ formally is a set of pairs in $\omega_{1} \times \omega$. $\left\langle\mathrm{L}_{\omega_{1}}[r], r\right\rangle \models \varphi$ iff $\varphi$ holds in $\mathrm{L}_{\omega_{1}}[r]$ with appearances of 
the predicate $\dot{r}$ in $\varphi$ interpreted by $r$.)

The number 2 in $G_{\mathrm{club}, 2}(\varphi)$ refers to the number of free variables in $\varphi$. Games $G_{\text {club }, k}(\varphi)$, for $k \neq 2$ in $\omega$ and $\varphi$ a formula with $k$ free variables, can defined similarly. All the definition can be relativized to a real $x$ by replacing $\mathrm{L}_{\omega_{1}}[r]$ with $\mathrm{L}_{\omega_{1}}[r, x]$ and letting $\varphi$ take $x$ as a parameter. They can be relativized to a set of reals $A$ by replacing $\mathrm{L}$ with $\mathrm{L}^{A}$ and allowing $\varphi$ to take an additional predicate interpreted by $A \cap \mathrm{L}_{\omega_{1}}^{A}[r]$.

The full iteration game on a countable model $M$ can be recast as a game of the form $G_{\text {club }, 2}(\varphi)$ relativized to a real coding $M$. Woodin [41] connects the determinacy of the games $G_{\mathrm{club}, k}(\varphi)$ and their relativizations to $\Sigma_{2}^{2}$ absoluteness under the combinatorial principle generic diamond $\left(\diamond_{G}\right)$. Determinacy for the games $G_{\text {club }, 2}(\varphi)$ is not provable from large cardinals, by Larson [17], but it may be provable from large cardinals and $\diamond_{G}$. Unfortunately the games are too strong to be handled by current methods in proofs of determinacy, precisely because they are strong enough to subsume the full iteration game. If there were a match for $G_{\text {club }, 2}$ similar to the matches in Theorems 2.4, 2.5, 3.2, 3.3, and 3.4, then the large cardinal involved would have to be stronger than a superstrong, far beyond the level of Woodin cardinals.

The following format produces a weaker game. Let $k<\omega$. Let $\vec{S}=\left\langle S_{a}\right|$ $\left.a \in\left[\omega_{1}\right]^{<k}\right\rangle$ be a collection of mutually disjoint stationary subsets of $\omega_{1}$, with a stationary set $S_{a}$ associated to each tuple $a \in\left[\omega_{1}\right]^{<k}$. Let $[\vec{S}]$ denote the set $\left\{\left\langle\alpha_{0}, \ldots, \alpha_{k-1}\right\rangle \in\left[\omega_{1}\right]^{k} \mid(\forall i<k) \alpha_{i} \in S_{\left\langle\alpha_{0}, \ldots, \alpha_{i-1}\right\rangle}\right\}$. Let $\varphi\left(x_{0}, \ldots, x_{k-1}\right)$ be a formula in $\mathcal{L}^{+}$. Define $G_{\omega_{1}, k}(\vec{S}, \varphi)$ to be the following game: Players I and II alternate playing $\omega_{1}$ natural numbers in the manner of Diagram 2, producing together a sequence $r \in \omega^{\omega_{1}}$. If there is a club $C \subset \omega_{1}$ so that $\left\langle\mathrm{L}_{\omega_{1}}[r], r\right\rangle \models \varphi\left[\alpha_{0}, \ldots, \alpha_{k-1}\right]$ for all $\left\langle\alpha_{0}, \ldots, \alpha_{k-1}\right\rangle \in[\vec{S}] \cap[C]^{k}$ then player I wins the run $r$. If there is a club $C \subset \omega_{1}$ so that $\left\langle\mathrm{L}_{\omega_{1}}[r], r\right\rangle \models \neg \varphi\left[\alpha_{0}, \ldots, \alpha_{k-1}\right]$ for all $\left\langle\alpha_{0}, \ldots, \alpha_{k-1}\right\rangle \in[\vec{S}] \cap[C]^{k}$ then player II wins $r$. If neither condition holds then both players lose.

Note that the two winning conditions in the definition of $G_{\omega_{1}, k}(\vec{S}, \varphi)$ cannot both hold. This uses the fact that each of the sets $S_{a}$ is stationary in $\omega_{1}$, and the demand in the conditions that $C$ must be club in $\omega_{1}$. Thus at most one player wins each run of $G_{\omega_{1}, k}(\vec{S}, \varphi)$. For $k>0$ it may well be that neither one of the winning conditions holds. So there may well be runs of $G_{\omega_{1}, k}(\vec{S}, \varphi)$ which are won by neither player. Determinacy for $G_{\omega_{1}, k}(\vec{S}, \varphi)$ is defined in the stronger of the two possible senses. The game is determined if one of the players has a winning strategy; a strategy which merely avoids losing is not enough.

Recall that a sharp for $\theta$ is an inner model $M$ with a final extender $E_{\text {top }}^{M}$, so that $E_{\text {top }}^{M}$ is an extender over $M$ and $M \models \theta$. Let $\theta$ be the sentence "crit $\left(E_{\text {top }}^{M}\right)$ is a Woodin cardinal". The minimal iterable sharp for $\theta$, if it exists, is denoted $0^{W}$. Recall that iterating out the top extender of a sharp $M$ produces a model $M^{*}$ and a club $I \subset \omega_{1}$ of indiscernibles for $M^{*}$, consisting of the images of $\operatorname{crit}\left(E_{\mathrm{top}}^{M}\right)$ under the iteration embeddings. In the case of $M=0^{W}$, the ordinals in $I$ are Woodin cardinals of $M^{*}$. The existence of $0^{W}$ thus implies the existence of an iterable model with a club of indiscernible Woodin cardinals, and in fact the two 
are equivalent.

Remark 3.7. Iterability for countable elementary substructures of $\mathrm{V}$ is not known at the level of $0^{W}$ - the strongest results in this direction are the ones of Neeman [31], reaching to the level of Woodin limits of Woodin cardinals - and the existence of $0^{W}$ is not known to follow from large cardinals in $\mathrm{V}$.

Theorem 3.8 (Neeman [35]). Suppose that $0^{W}$ exists. Then the games $G_{\omega_{1}, k}(\vec{S}, \varphi)$ are determined, for all $\vec{S}, k$, and $\varphi$.

There are two parameters determining the payoff of the game $G_{\omega_{1}, k}(\vec{S}, \varphi)$. One is the formula $\varphi$ and the number $k$ of its free variables. The other is the sequence $\vec{S}$. The formula $\varphi$, or the formula $\varphi$ and the real $x$ in the case of games relativized to a real, is the definable part of the payoff condition, analogous to the $<\omega^{2}-\Pi_{1}^{1}$ set, or more precisely to its recursive definition, in Theorems 3.2, 3.3, and 3.4. The sequence $\vec{S}$ consists of disjoint stationary sets, and this makes it highly nondefinable. It has no parallel in Theorems 3.2, 3.3, and 3.4. It is necessary in Theorem 3.8, and the winning strategy in $G_{\omega_{1}, k}(\vec{S}, \varphi)$ depends on $\vec{S}$. But which of the players has a winning strategy is determined independently of $\vec{S}$ :

Theorem 3.9 (Neeman [35]). Suppose that $0^{W}$ exists. Let $\vec{S}=\left\langle S_{a} \mid a \in\left[\omega_{1}\right]^{<k}\right\rangle$ and $\vec{S}^{*}=\left\langle S_{a}^{*} \mid a \in\left[\omega_{1}\right]^{<k}\right\rangle$ be two sequences of mutually disjoint stationary subsets of $\omega_{1}$. Then player I (respectively II) has a winning strategy in $G_{\omega_{1}, k}(\vec{S}, \varphi)$ iff she has a winning strategy in $G_{\omega_{1}, k}\left(\vec{S}^{*}, \varphi\right)$.

Define $\partial_{\omega_{1}}(k, \varphi)$ to be 1 if player I has a winning strategy in $G_{\omega_{1}, k}(\vec{S}, \varphi)$ for some, and using Theorem 3.9 equivalently for all, $\vec{S}$. Define $\partial_{\omega_{1}}(k, \varphi)$ to be equal to 0 otherwise.

Theorem 3.10 (Neeman [35]). Suppose that $0^{W}$ exists. Then $0^{W}$ and $\{\langle k, \varphi\rangle \mid$ $\left.\partial_{\omega_{1}}(k, \varphi)=1\right\}$ are each recursive in the other.

Theorems 3.8 and 3.10 establish the same precise connection between $0^{W}$ and games of length $\omega_{1}$ that exists between $0^{\sharp}$ and $<\omega^{2}-\Pi_{1}^{1}$ games of length $\omega$. They provide another step, the first to reach games of length $\omega_{1}$, in the project of matching the hierarchy of large cardinals with the hierarchy of long games.

\section{References}

[1] John W. Addison and Yiannis N. Moschovakis. Some consequences of the axiom of definable determinateness. Proc. Nat. Acad. Sci. U.S.A., 59:708-712, 1968.

[2] David Blackwell. Infinite games and analytic sets. Proc. Nat. Acad. Sci. U.S.A., 58:1836-1837, 1967.

[3] Morton Davis. Infinite games of perfect information. In Advances in game theory, pages 85-101. Princeton Univ. Press, Princeton, N.J., 1964. 
[4] David Gale and Frank M. Stewart. Infinite games with perfect information. In Contributions to the theory of games, vol. 2, Annals of Mathematics Studies, no. 28, pages 245-266. Princeton University Press, Princeton, N. J., 1953.

[5] Leo Harrington. Analytic determinacy and $0^{\sharp}$. J. Symbolic Logic, 43(4):685-693, 1978.

[6] Greg Hjorth. A boundedness lemma for iterations. J. Symbolic Logic, 66(3):10581072, 2001.

[7] Steve Jackson. Structural consequences of AD. In Handbook of Set Theory. To appear.

[8] R. Björn Jensen. The fine structure of the constructible hierarchy. Ann. Math. Logic, 4:229-308; erratum, ibid. 4 (1972), 443, 1972. With a section by Jack Silver.

[9] Alexander S. Kechris. AD and projective ordinals. In Cabal Seminar 76-77 (Proc. Caltech-UCLA Logic Sem., 1976-77), volume 689 of Lecture Notes in Math., pages 91-132. Springer, Berlin, 1978.

[10] Alexander S. Kechris and Donald A. Martin, editors. Cabal Seminar 77-79, volume 839 of Lecture Notes in Mathematics, Berlin, 1981. Springer.

[11] Alexander S. Kechris, Donald A. Martin, and Yiannis N. Moschovakis, editors. Cabal seminar 79-81, volume 1019 of Lecture Notes in Mathematics, Berlin, 1983. SpringerVerlag.

[12] Alexander S. Kechris, Donald A. Martin, and John R. Steel, editors. Cabal Seminar 81-85, volume 1333 of Lecture Notes in Mathematics, Berlin, 1988. Springer-Verlag.

[13] Alexander S. Kechris and Yiannis N. Moschovakis, editors. Cabal Seminar 76-77, volume 689 of Lecture Notes in Mathematics, Berlin, 1978. Springer.

[14] Alexander S. Kechris and W. Hugh Woodin. Equivalence of partition properties and determinacy. Proc. Nat. Acad. Sci. U.S.A., 80(6 i.):1783-1786, 1983.

[15] Kenneth Kunen. Some applications of iterated ultrapowers in set theory. Ann. Math. Logic, 1:179-227, 1970.

[16] Casimir Kuratowski. Sur les théorèmes de séparation dans la Théorie des ensembles. Fund. Math., 26:183-191, 1936.

[17] Paul Larson. The canonical function game. To appear.

[18] Donald A. Martin. The axiom of determinateness and reduction principles in the analytical hierarchy. Bull. Amer. Math. Soc., 74:687-689, 1968.

[19] Donald A. Martin. Measurable cardinals and analytic games. Fund. Math., 66:287291, 1969/1970.

[20] Donald A. Martin. Borel determinacy. Ann. of Math. (2), 102(2):363-371, 1975.

[21] Donald A. Martin. The largest countable this, that, and the other. In Cabal seminar 79-81, volume 1019 of Lecture Notes in Math., pages 97-106. Springer, Berlin, 1983.

[22] Donald A. Martin and John R. Steel. A proof of projective determinacy. J. Amer. Math. Soc., 2(1):71-125, 1989.

[23] Donald A. Martin and John R. Steel. Iteration trees. J. Amer. Math. Soc., 7(1):1-73, 1994.

[24] William J. Mitchell. Sets constructible from sequences of ultrafilters. J. Symbolic Logic, 39:57-66, 1974. 
[25] William J. Mitchell. Sets constructed from sequences of measures: revisited. J. Symbolic Logic, 48(3):600-609, 1983.

[26] William J. Mitchell and John R. Steel. Fine structure and iteration trees, volume 3 of Lecture Notes in Logic. Springer-Verlag, Berlin, 1994.

[27] Yiannis N. Moschovakis. Descriptive set theory, volume 100 of Studies in Logic and the Foundations of Mathematics. North-Holland Publishing Co., Amsterdam, 1980.

[28] Jan Mycielski and Hugo Steinhaus. A mathematical axiom contradicting the axiom of choice. Bull. Acad. Polon. Sci. Sér. Sci. Math. Astronom. Phys., 10:1-3, 1962.

[29] Jan Mycielski and Stanisław Świerczkowski. On the Lebesgue measurability and the axiom of determinateness. Fund. Math., 54:67-71, 1964.

[30] Itay Neeman. Optimal proofs of determinacy. Bull. Symbolic Logic, 1(3):327-339, 1995.

[31] Itay Neeman. Inner models in the region of a Woodin limit of Woodin cardinals. Ann. Pure Appl. Logic, 116(1-3):67-155, 2002.

[32] Itay Neeman. Optimal proofs of determinacy. II. J. Math. Log., 2(2):227-258, 2002.

[33] Itay Neeman. The determinacy of long games, volume 7 of de Gruyter Series in Logic and its Applications. Walter de Gruyter GmbH \& Co. KG, Berlin, 2004.

[34] Itay Neeman. Determinacy for games ending at the first admissible relative to the play. To appear.

[35] Itay Neeman. Games of length $\omega_{1}$. To appear.

[36] Itay Neeman. Inner models and ultrafilters in $L(\mathbb{R})$. To appear.

[37] John C. Oxtoby. The Banach-Mazur game and Banach category theorem. In Contributions to the theory of games, vol. 3, Annals of Mathematics Studies, no. 39, pages 159-163. Princeton University Press, Princeton, N. J., 1957.

[38] John R. Steel and Robert Van Wesep. Two consequences of determinacy consistent with choice. Trans. Amer. Math. Soc., 272(1):67-85, 1982.

[39] W. Hugh Woodin. Some consistency results in ZFC using AD. In Cabal seminar 79-81, volume 1019 of Lecture Notes in Math., pages 172-198. Springer, Berlin, 1983.

[40] W. Hugh Woodin. Supercompact cardinals, sets of reals, and weakly homogeneous trees. Proc. Nat. Acad. Sci. U.S.A., 85(18):6587-6591, 1988.

[41] W. Hugh Woodin. Beyond $\sum_{\sim}^{2}$ absoluteness. In Proceedings of the International Congress of Mathematicians, Vol. I (Beijing, 2002), pages 515-524, Beijing, 2002. Higher Ed. Press.

[42] Martin Zeman. Inner models and large cardinals, volume 5 of de Gruyter Series in Logic and its Applications. Walter de Gruyter \& Co., Berlin, 2002.

Department of Mathematics

University of California Los Angeles

Los Angeles, CA 90095-1555

E-mail: ineeman@math.ucla.edu 